\title{
Shu-Yu capsule, a Traditional Chinese Medicine formulation, attenuates premenstrual syndrome depression induced by chronic stress constraint
}

\author{
XINGXIAO GAO ${ }^{1}$, PENG SUN ${ }^{1}$, MINGQI QIAO ${ }^{1,2}$, SHENG WEI $^{1}$, LING XUE $^{1}$ and HUIYUN ZHANG ${ }^{1}$ \\ ${ }^{1}$ Lab of Traditional Chinese Medicine Classical Theory, Ministry of Education; \\ ${ }^{2}$ Institute of Traditional Chinese Medicine Theory, School of Basic Medicine, \\ Shandong University of Traditional Chinese Medicine, Jinan, Shandong 250355, P.R. China
}

Received August 26, 2013; Accepted April 4, 2014

DOI: $10.3892 / \mathrm{mmr} .2014 .2599$

\begin{abstract}
The present study aimed to investigate the therapeutic effect of the Shu-Yu capsule (SYC), a Traditional Chinese Medicine formulation, on premenstrual syndrome (PMS) depression and the ratio of glutamate (Glu) to $\gamma$-aminobutyric acid (GABA) in a rat model of PMS depression. Rats were randomly divided into a control group, model group, fluoxetine group, SYC group and saikosaponins (SS) group. The therapeutic effect of SYC was evaluated using a sucrose preference test (SPT), open field test (OFT) and body mass following four days of treatment. Hippocampal extracellular fluid was collected by microdialysis and the levels of Glu and GABA in the microdialysate were measured by high performance liquid chromatography. The results revealed that, compared with the control group, the sucrose preference coefficient (SC\%), total score of OFT and body mass of the model group were significantly lower $(\mathrm{P}<0.01, \mathrm{P}<0.05$ and $\mathrm{P}<0.01$, respectively). However, the sucrose preference coefficient, total score of OFT and body mass of the SYC group were all significantly increased compared with the model group $(\mathrm{P}<0.05)$. Furthermore, SYC inhibited the decrease of the Glu/GABA ratio in the hippocampus of rats with PMS depression. It was concluded that SYC effectively improved the symptoms of PMS depression, possibly by inhibiting the
\end{abstract}

Correspondence to: Dr Mingqi Qiao, Institute of Traditional Chinese Medicine Theory, School of Basic Medicine, Shandong University of Traditional Chinese Medicine, Building No. 2, No. 4655 Daxue Road, Jinan, Shandong 250355, P.R. China E-mail: 15965611380@163.com

Abbreviations: SYC, Shu-Yu capsule; PMS, premenstrual syndrome; Glu, glutamate; GABA, $\gamma$-aminobutyric acid; SPT, sucrose preference test; OFT, open field test; $\mathrm{SC} \%$, sucrose preference coefficient; HPLC, high performance liquid chromatography.

Key words: premenstrual syndrome depression, Shu-Yu capsule, $\gamma$-aminobutyric acid, glutamate dysregulation of the Glu/GABA balance in the central nervous system.

\section{Introduction}

Premenstrual syndrome (PMS) is a poorly understood disorder characterized by the cyclical occurrence of behavioral, psychiatric and physical symptoms during the premenstrual phase or late luteal phase of the menstrual cycle. In a number of cases, females with PMS suffer from irritability, tension and depression so severe that their personal and professional lives are frequently affected (1). Symptom relief is the goal for the treatment of PMS and it is generally acknowledged that serotonergic antidepressants, including citalopram, escitalopram, fluoxetine, sertraline and venlafaxine, are the first-line pharmacological therapies (2-4). However, these treatments are limited by their cost and the severe side effects associated with long-term medication regimens (5-7). Therefore, studies to develop novel pharmacological agents with improved therapeutic effects and fewer side effects are highly important.

Shu-Yu capsule (SYC), a novel Chinese materia medica preparation, has been developed by our research team based on years of experience in the treatment of PMS and depression. SYC is composed of active ingredients extracted from four Chinese herbs, namely bupleurum chinense, white paeony root, radix glycyrrhizae and rhizoma cyperi. The notable efficacy of SYC in the treatment of PMS and improvement of depressive symptoms has been confirmed in clinical trials. However, the underlying mechanisms of these effects remain to be elucidated. In our previous studies, the effects of SYC were studied in rats with depression $(8,9)$. However, the survival environment of living brain cells was not observed, and dynamic changes of brain neurotransmitters in real time were not monitored in these studies. To overcome these limitations, a combination of microdialysis and high performance liquid chromatography (HPLC) analysis was adopted in the present study. These methods allowed the dynamic analysis of glutamate (Glu) and $\gamma$-aminobutyric acid (GABA) levels in the hippocampus of rats with PMS depression exposed to SYC.

Although the underlying pathophysiological changes have yet to be delineated, numerous hypotheses on the recurrence 
of PMS depression have been proposed $(10,11)$. The two most intensively studied and relevant neurotransmitter systems implicated in the genesis of PMS symptoms are the GABAergic and serotonergic systems (12). It has been confirmed that there is a subtle excitation/inhibition balance in the neural network. Neurotransmitter systems maintain a steady-state balance by fine-tuning of the neurosecretory network. Disruptions to this steady-state equilibrium defines the aetiology of numerous neurological disorders, including anxiety, depression and biphasic affective disorders (13-15). Glu and GABA are the main excitatory (EAA) and inhibitory (IAA) amino acids in the central nervous system (CNS), respectively. The balance between the two neurotransmitter systems is essential to the maintenance of normal brain function $(16,17)$. To a certain extent, the ratio of Glu to GABA reflects the functional status of the brain (18). Preclinical and clinical studies have provided increasing amounts of data on the effects of amino acid neurotransmitters on the neurobiology of mood disorders. Therefore, GABA and Glu systems may be potential therapeutic targets in depression and mood disorders (19-21). It has been identified that Glu levels alter throughout the menstrual cycle in all females and that PMS patients are more sensitive to such changes (22). Furthermore, it has been reported that the symptoms of premenstrual dysphoric disorder (PMDD) patients were closely associated with the levels of GABA and the sensitivity of GABA receptors in the luteal phase (23-25).

In the present study, the therapeutic effects of SYC on PMS depression were investigated in a rat model of PMS depression. The rat models of PMS depression were induced by chronic stress constraint and the subsequent changes in the ratio of Glu to GABA were also observed.

\section{Materials and methods}

Reagents. L-glutamate, $\gamma$-aminobutyric acid and $\beta$-mercaptoethanol were provided by Sigma (St. Louis, MO, USA). Methanol was purchased from Tianjin four Friends of the Fine Chemicals Co., Ltd. (Tianjin, China) and $\mathrm{KH}_{2} \mathrm{PO}_{4}, \mathrm{NaOH}$ and $\mathrm{Na}_{2} \mathrm{~B}_{4} \mathrm{O}_{7} \times 10 \mathrm{H}_{2} \mathrm{O}$ were purchased from Tianjin Dengke Chemical Reagents Co., Ltd. (Tianjin, China). Salt-cure tooth acrylic resin and self-cure denture base material liquid were supplied by Shanghai New Century Dental Material Co., Ltd. (Shanghai, China). Gentamicin sulfate and sodium chloride for injection were obtained from Shandong Lukang Chen Xin Pharmaceutical Co., Ltd. (Shandong, China).

Composition and preparation of SYC. SYC is novel Chinese materia medica preparation developed by our research team and was commissioned to Haichuan Medicine Manufacturer (Qingdao, China) for production. SYC is has four major active ingredients (saikosaponin A, albiflorin, volatile oil of cyperus rotundus and glycyrrhizic acid) extracted from bupleurum chinense, white paeony root, radix glycyrrhizae and rhizoma cyperi, respectively. The above four components were extracted separately with $60 \%$ (or $70 \%$ ) ethanol or deionized water from the raw herbs. The resulting extracts were then subjected to macroporous resin separation (or acid precipitation) and the eluents from each extract were concentrated and dried under reduced pressure to obtain the corresponding dry powder. In view of its volatility, volatile oil extracted from rhizoma cyperi was converted into $\beta$-cyclodextrin prior to drying. Finally, the powders were mixed with calcium bicarbonate (acting as anti-absorbent agents), granulated and dispensed into the capsule.

Animals and treatments. Thirty female Wistar rats (weight, 200-220 g) were supplied by the Center for Laboratory Animals of Shandong University of Traditional Chinese Medicine (Shandong, China). The rats were kept in conventional plastic cages $(43 \times 30 \times 19 \mathrm{~cm})$ with a standard rat chow diet and maintained on a standard 12-h light/dark cycle, with lights on at 8:00 am. The ambient temperature was controlled at $23 \pm 1^{\circ} \mathrm{C}$ and humidity was maintained at $55 \pm 5 \%$. All of the animals were allowed to adjust to the environment for one week prior to the start of the experiment. All experiments were conducted in accordance with the revised 1996 laws and regulations of the National Institute of Health Guide for the Care and Use of Laboratory Animals, and the study was approved by the ethics committee of Shandong University of Traditional Chinese Medicine (Jinan, China). All procedures were conducted in a manner that minimized pain and suffering for the animals.

Healthy and not-pregnant female rats $(n=30)$ with regular estrous cycles were used in the present study. They were divided into five groups $(n=6)$, including the control group, model group, fluoxetine group, SYC group and saikosaponins (SS) group. The PMS depression model was established in rats of the model group, fluoxetine group, SYC group and SS group. Rats that were in the non-acceptance phase (NR) of the estrous cycle were enrolled into the modeling course (four days of two continuous non-acceptance phases; Fig. 1). Briefly, the rats' forelegs and opposite-side metapedes were bound with asepsis absorbent gauzes to restrict free movement, while still allowing the ability to access food and water (26).

Rats of the control group and the model group were gavaged with sterile water at a dose of $10 \mathrm{ml} \mathrm{kg}^{-1}$ of body weight. Rats in the SYC group received SYC at a dose of $408 \mathrm{mg} \mathrm{kg}^{-1}$ of body weight by intragastric administration. Rats in the SS group were gavaged daily with saikosaponin extract at a dose of $0.72 \mathrm{mg} \mathrm{kg}^{-1}$. Rats in the fluoxetine group were gavaged daily with fluoxetine hydrochloride at a dose of $2.67 \mathrm{mg} \mathrm{kg}^{-1}$. All animals were treated every day from 8:30-9:00 am. The treatments lasted for four days in two continuous non-acceptance phases.

Evaluation methods for rat model and clinical efficacy of drugs

Vaginal smear. The estrous cycles of rats were confirmed by observing the shape of vaginal cells (26). The specific procedure of vaginal smear was as follows: A small quantity of isotonic sodium chloride was drawn by a dropper and was dropped repeatedly into a rat's vagina two to three times. The suction fluid was over-laid on a glass slide to be naturally air-dried and then the cell appearance was observed under a CX21BIM-SET5 microscope (Olympus Corporation, Tokyo, Japan). Based on the number of white blood cells in the vaginal smear, the estrous cycle was divided into the acceptance (pre-estrous and estrous) and non-acceptance period (meto-estrous and anestrous). Vaginal smears were conducted three times every day for seven days to confirm the estrous cycle of every experimental rat.

Open field test $(O F T)$. The OFT was performed with a wooden and uncowled box $(100 \times 100 \times 50 \mathrm{~cm})$. The sides and 
undersurfaces were black. The undersurface was partitioned into 25 areas of the same size by white lines. Each rat was placed in the center of the experimental apparatus prior to testing and was allowed to explore it for three minutes. The crossing score and rearing score were automatically recorded with a photographic recording system (27). The crossing score was the number of times that the animals crossed the undersurface grilles, while the rearing score was the number of times that the animals made a perpendicular act. The perpendicular act was defined as standing on hind legs with paws pressed against the arena wall, sniffing continuously for at least $2 \mathrm{sec}$ (28). The sum of crossing and rearing scores was recorded as the total score.

Sucrose preference test (SPT). The SPT was performed as described previously (29). During the testing period, rats were allowed a free choice between two bottles for $24 \mathrm{~h}$. One bottle contained $1 \%$ sucrose solution and the other contained sterile water. To exclude the possible effects of side preference in drinking, bottle positions were switched following $12 \mathrm{~h}$. The consumption of sterile water (W1) and sucrose solution (W2) was measured by weighing the bottles. Sucrose preference was calculated from the consumed amount of sucrose solution, which was expressed as the percentage of the total amount of liquid consumed (SC\%); SC\%=W2 (g)/[W2 (g) + W1 (g)]x100\%.

Surgery and microdialysis. Rats were anesthetized with $1 \%$ sodium pentobarbital (40 45 mg kg-1 $4 \mathrm{ml} \mathrm{kg}^{-1}$, administered intraperitoneally) prior to stereotaxic surgery. A hole for the cannula was drilled into the skull. A guide cannula (CMA-12; CMA Microdialysis AB, Acton, MA, USA) was implanted bilaterally into the right hippocampus of the rats using the following coordinates: AP, $5.8 \mathrm{~mm}$; ML, $4.6 \mathrm{~mm}$ from bregma; DV $7.6 \mathrm{~mm}$ from dura according to the previously described methods (30). A mound of cranioplastic cement was formed around the cannula to fix the guide cannula and a stylus was inserted to maintain patency. Immediately following completion of the surgery, a gentamycin sulfate injection $\left(1 \mathrm{ml} \mathrm{kg}^{-1}\right.$, administered subcutaneously) was administered at the cervical part of the rats. Then the rats were individually housed with intensive care until they recovered from surgery.

Animals were allowed to recover for a minimum of three days. In the morning prior to microdialysis, the rats were examined by a vaginal smear to determine which estrous cycle they were in, NR or reception period (R). A 2 mm CMA-12 probe (CMA Microdialysis AB, Kista, Sweden) was placed into the right hippocampus vertically. Artificial cerebrospinal fluid (artCSF) including $147 \mathrm{mM} \mathrm{NaCl}, 1.26 \mathrm{mM} \mathrm{CaCl}_{2}, 2.5 \mathrm{mM}$ $\mathrm{KCl}$ and $1.18 \mathrm{mM} \mathrm{MgCl}_{2}$ in sterile water was perfused through the probe at a rate of $2.5 \mu \mathrm{l} \mathrm{min}{ }^{-1}$ with a CMA microdialysis syringe pump. Samples of each rat were collected four times ( $R$ and NR prior to modeling, R and NR following modeling), with continuous sampling for $3 \mathrm{~h}$ each time.

High performance liquid chromatography (HPLC). The concentration of GABA and Glu was analyzed using an Agilent 1100 series HPLC system with a G1321A fluorimetric detector (Agilent Technologies GmbH, Waldbronn, Germany) and a Kromasil 100-5-C18 4.6x250 mm column ( $5 \mu \mathrm{m}$; AkzoNobel, Brewster, NY, USA). Briefly, $20 \mu 1$ dialysate was mixed with $10 \mu 1$ derivatization agent $(26.8 \mathrm{mg} o$-phthalaldehyde was dissolved in $2 \mathrm{ml}$ dehydrated ethanol, and then $8 \mathrm{ml} 0.1 \mathrm{M}$ borate buffer and $40 \mu 1 \beta$-mercaptoethanol were added) and left on the bench for 2 min for precolumn derivatization reaction. The mobile phase was composed of $45 \%$ potassium dihydrogen phosphate (0.1 M, pH 6.7) (Tianjing Damao Chemical Reagent Factory, Tianjin, China) and 55\% methanol (Tianjin Shield Fine Chemical Product Co. Ltd., Tianjin, China). The excitation and the emission spectrums of the fluorescence detector (FLD) were $340 \mathrm{~nm}$ and $455 \mathrm{~nm}$, respectively. The flow rate was fixed at $1.0 \mathrm{ml} \mathrm{min}{ }^{-1}$ with an injection volume of $20 \mu 1$ at a column temperature of $40^{\circ} \mathrm{C}$.

Statistical analysis. GraphPad Prism Medical graphics software, version 5 (GraphPad Software, Inc., La Jolla, CA, USA) was used for data analysis. Two-way analysis of variance (ANOVA) with bonferroni's post-hoc tests were used to determine statistical differences, with treatment as the main factor and time as the repeated factor. $\mathrm{P}<0.05$ was considered to indicate a statistically significant difference.

\section{Results}

Experimental procedures. The major experimental stages are presented in Fig. 1. All animals were allowed to adjust to the environment for one week prior to the experiment, followed by one week of vaginal smear to confirm the estrous cycle of each rat. The stereotactic surgery was performed on the first day of a certain non-acceptance period. All rats were given post-operative care and normal feeding to ensure their recovery from surgery. Following three days, the acceptance period (R1) and non-acceptance period sampling (NR1) began, followed by model preparation (Modeling) and drug intervention stages. Finally, microdialysis sampling was performed in R1 and NR1 groups.

Effect of SYC on body mass in rats with PMS depression. To quantify the recovery conditions of rats following surgical operation and the effect of SYC, their body mass was determined at several time-points, including pre-surgery, post-surgery, pre-modeling and post-modeling. The changes in body mass were described by the body mass gain coefficient (GC\%). The GC\% was calculated with the following formula: GC\%=(M2-M1)/M1 (M1 represents the body mass of the former stage, while M2 represents that of the later stage). As illustrated in Fig. 2, there were no significant differences in the GC\% among the five groups in stages of pre-surgery, post-surgery and pre-modeling. However, in the post-modeling stage, there were differences in the $\mathrm{GC} \%$ among the five groups. The GC\% of the SS group was similar to that of the control group, but the GC\% of the model group was significantly lower than that of the control group $(\mathrm{P}<0.01)$. Meanwhile, the GC\% of the SYC and fluoxetine groups was significantly higher than that of the model group $(\mathrm{P}<0.05)$. These results suggested that SYC attenuated the body mass reduction in rats.

Effect of SYC on OFT and SPT in rats with PMS depression. OFT was performed as a behavioral assessment method for the measurement of PMS depression. The crossing and rearing scores were recorded in the pre-modeling and post-modeling stages. Total scores of OFT were defined as the sum of crossing and rearing score. As demonstrated in Fig. 3A, the total scores of OFT were not significantly different among the 


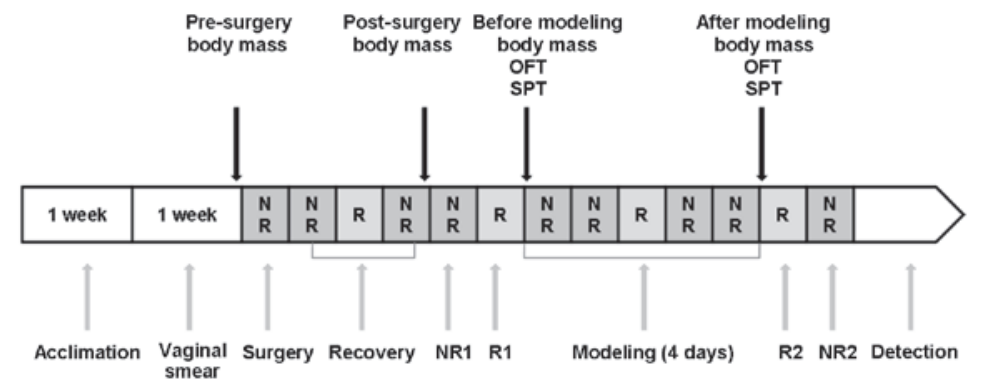

Figure 1. Schematic of experimental procedures. All animals were allowed to adjust to the environment for one week prior to the start of the experiment. In the next week, vaginal smears were conducted to confirm the estrous cycle of every experimental rat. Each rectangle filled with gray color represents a day. SPT, OFT, body mass weighing, sampling, modeling and drug interference were conducted accordingly. R1, reception phase prior to modeling; NR1, non-reception phase prior to modeling; R2, reception phase following modeling; NR1, non-reception phase following modeling; OFT, open field test; SPT, sucrose preference test.

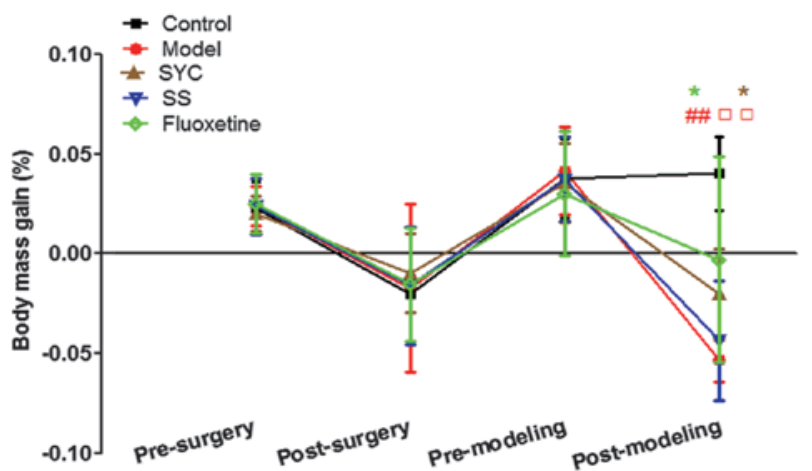

Figure 2. Changes in the body mass of five groups in different periods. Body mass was weighed at stages of pre-surgery, post-surgery, pre-modeling and post-modeling. GC\% was calculated according to the following formula: $\mathrm{GC} \%=(\mathrm{M} 2-\mathrm{M} 1) / \mathrm{M} 1$ (M1 represents the body mass of the former stage, while $\mathrm{M} 2$ for the later stage). ${ }^{\# \#} \mathrm{P}<0.01$, pre-modeling vs post-modeling in model group; ${ }^{\mathrm{P}}<0.05$, SYC group or fluoxetine group vs model group in post-modeling stage; ${ }^{\square} \mathrm{P}<0.01$, model group vs control in post-modeling stage. SYC, Shu-yu capsule group; SS, saikosaponins group; GC\%, body mass gain coefficient.

five groups at the stage of pre-modeling $(\mathrm{P}>0.05)$. Following four-day modeling, total scores of the model group in the post-modeling stage were significantly lower than those in the pre-modeling stage $(\mathrm{P}<0.05)$. Total scores of the model group in the post-modeling stage were also significantly lower than those of the control group at the post-modeling stage $(\mathrm{P}<0.01)$. In addition, after four-day modeling, total scores of the SYC and fluoxetine groups were significantly higher than those of the model group $(\mathrm{P}<0.05)$; however, there was no significant difference between the OFT total scores of the SS group and the model group $(\mathrm{P}>0.05)$.

SPT, the most commonly recognized method for simulating the core symptoms of depression-anhedonia, was performed to evaluate the PMS depression model in the present study. SPT was performed in the pre-modeling and post-modeling stages. The sucrose preference coefficient was expressed as the percentage of the total amount of consumed liquid (SC\%). As demonstrated in Fig. 3B, there were no significant differences in the $\mathrm{SC} \%$ among the five groups at the stage of pre-modeling ( $\mathrm{P}>0.05$ ). Following four-day modeling, the SC\% of the model group decreased. Compared with that of the pre-modeling stage, the $\mathrm{SC} \%$ of the model group in the post-modeling stage was significantly lower $(\mathrm{P}<0.01)$. However, in the post-modeling
A

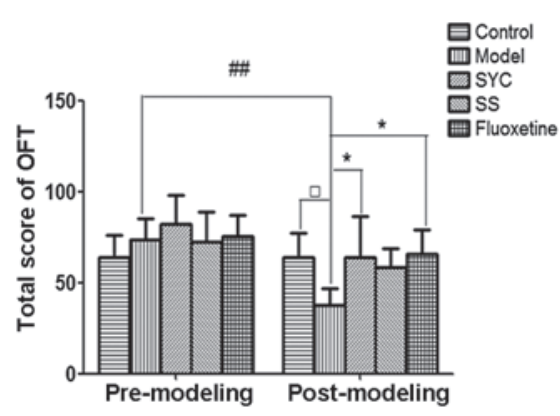

B

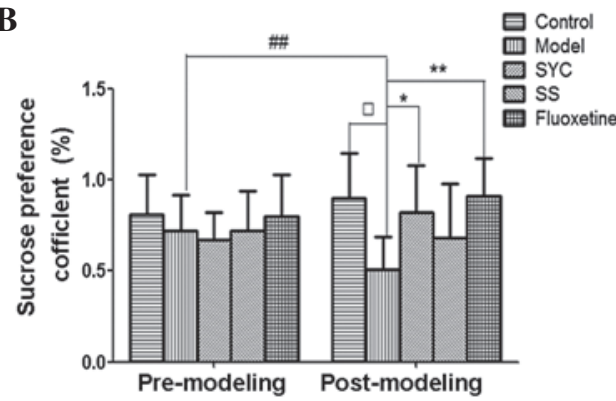

Figure 3. Changes in the total scores of OFT and sucrose preference coefficient (SC\%) of SPT of five groups in different periods. OFT and SPT were performed in the pre- and post-modeling stages. (A) OFT results of the five groups in the pre- and post-modeling stage. Total scores of OFT were defined as the sum of crossing and rearing score. (B) SPT results of the five groups in the pre- and post-modeling stages. The sucrose preference coefficient was expressed as the percentage of the total amount of consumed liquid (SC\%). ${ }^{\#} \mathrm{P}<0.05,{ }^{\# \#} \mathrm{P}<0.01$, pre-modeling vs post-modeling in model group; ${ }^{*} \mathrm{P}<0.05$, SYC group or fluoxetine group vs model group in post-modeling stage; ${ }^{\square} \mathrm{P}<0.05,{ }^{\square} \mathrm{P}<0.01$, model group vs control in post-modeling stage. OFT, open field test; SPT, sucrose preference test; SYC, Shu-yu capsule group; SS, saikosaponins group.

stage, the SC\% of the control, SYC and fluoxetine groups were all significantly higher than those of the model group $(\mathrm{P}<0.05)$, while the SC\% of the SS group demonstrated no significant difference compared with the model group $(\mathrm{P}>0.05)$. These results suggested that the PMS depression model was successfully established and that SYC attenuated PMS depression in these rats.

Ratio of Glu to GABA in the right hippocampus during different estrous cycles. Since there is a delicate excitation/inhibition balance between Glu and GABA in the CNS and it is possible that they may have indispensable roles in the 
A

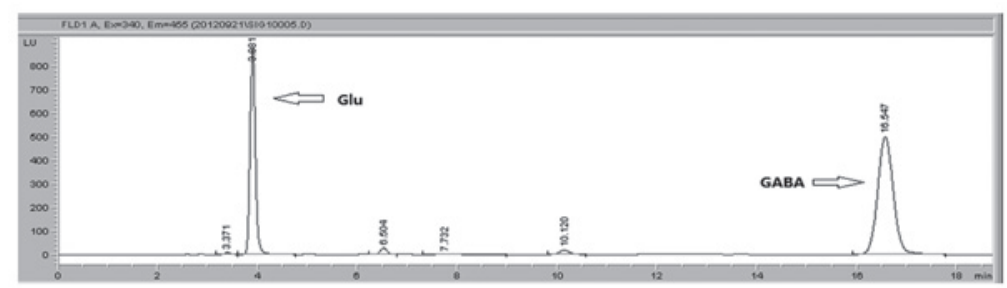

B

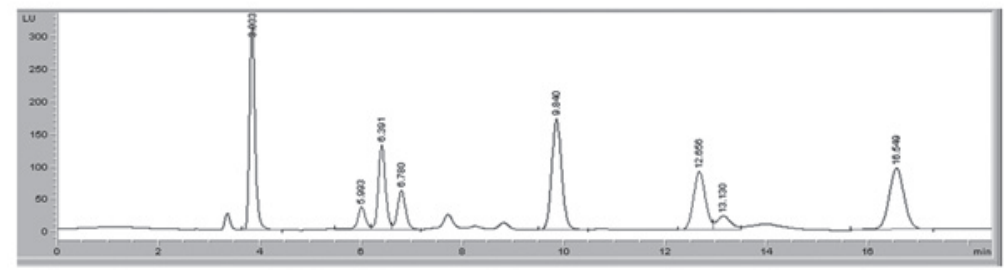

Figure 4. Changes in Glu and GABA levels in the right hippocampus of rats with PMS depression. Glu and GABA levels were detected by HPLC analysis. (A) HLPC results of mixed standard solution $(10 \mu \mathrm{g} / \mathrm{ml})$. (B) HLPC results of the mircodialysis sample. Glu, glutamate; GABA, $\gamma$-aminobutyric acid; PMS, premenstrual syndrome; HPLC, high performance liquid chromatography.
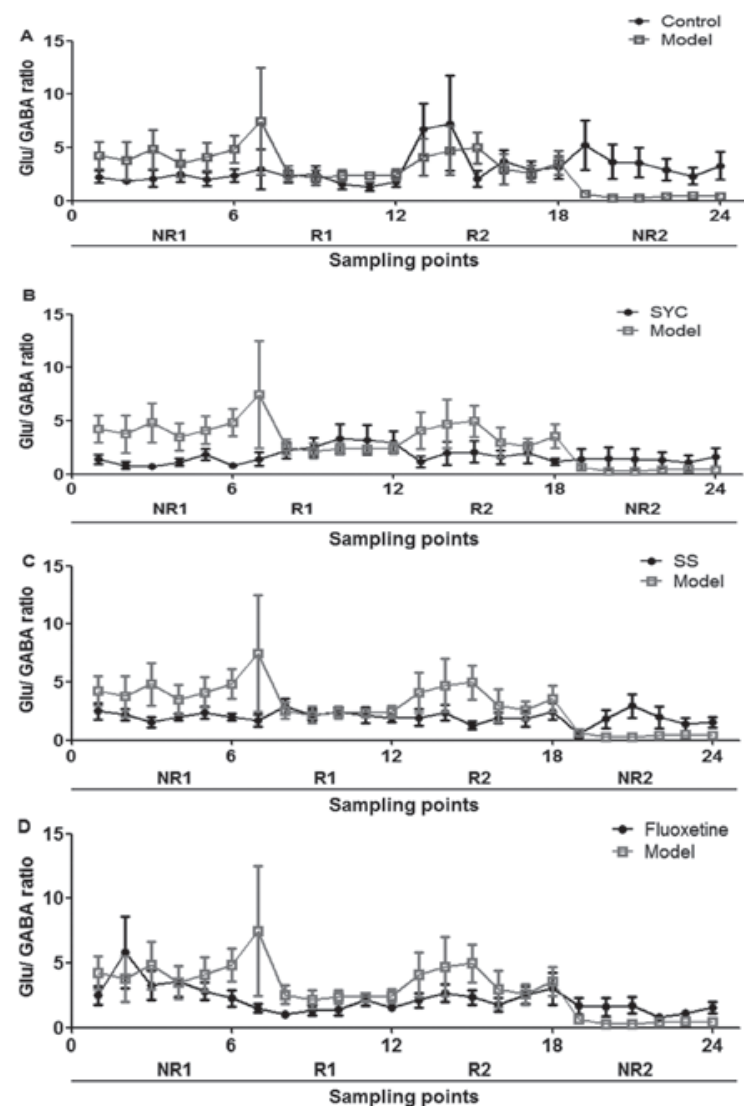

Figure 5. Ratios of Glu to GABA at different sampling points of different estrous cycles. (A) Comparison of the Glu/GABA ratio between the model and control groups. (B) Comparison of the Glu/GABA ratio between the model and SYC groups. (C) Comparison of Glu/GABA ratio between the model and SS groups. (D) Comparison of the Glu/GABA ratio between the model and fluoxetine groups. 1 24 represent 24 sampling points with six points in each; $\mathrm{R} 1$, reception phase prior to modeling; NR1, non-acceptance period prior to modeling; R2, acceptance period following modeling; NR2, non-acceptance period following modeling. Glu, glutamate; GABA, $\gamma$-aminobutyric acid; SYC, Shu-yu capsule group; SS, saikosaponins group.

etiology of PMS, the ratio of Glu to GABA was investigated. HPLC analysis was conducted to detect the levels of Glu and



Figure 6. Ratios of Glu to GABA of all the groups in different estrous cycles. Differences among the groups in values of Glu/GABA ratios in the stages of $\mathrm{NR} 1, \mathrm{R} 1$ and $\mathrm{R} 2$ were analyzed by two-factor analysis of variance. ${ }^{\# \#} \mathrm{P}<0.001$, NR2 vs NR1 in model group; ${ }^{*} \mathrm{P}<0.05,{ }^{* *} \mathrm{P}<0.01, \mathrm{SYC}$ or fluoxetine group vs model group in NR2 stage; $\square \mathrm{P}<0.01$, model group vs control in NR2 stage. R1, reception phase prior to modeling; NR1, non-acceptance period prior to modeling; R2, acceptance period following modeling; NR2, non-acceptance period following modeling. Glu, glutamate; GABA, $\gamma$-aminobutyric acid; SY, Shu-yu.

GABA in the microdialysis samples. Microdialysis samples were collected at four estrous cycles (NR1, R1, R2 and NR2), with six sampling time-points (every $0.5 \mathrm{~h}$ between 9:00-12:00 am) in the same estrous cycle. As demonstrated in Fig. 4, the residence time for Glu and GABA was 3.9 and $16.5 \mathrm{~min}$, respectively.

Following this, the ratio of Glu to GABA was calculated. The comparison of the Glu/GABA ratios between the modeling group and the control, SYC, SS and fluoxetine groups are illustrated in Fig. 5. As shown in Fig. 5A, the Glu/GABA ratios in the stages of NR1, R1 and R2 were not evidently different between the model and the control groups. However, the Glu/GABA ratios in the model group were lower than those in the control group at point 18-24 during NR2. The fluctuation of Glu/GABA ratios in the SYC, fluoxetine and SS groups was consistent with that in the control group in the NR1, R1 and R2 stages. However, in the NR2 stage, the Glu/GABA ratios of the above four groups were all higher than those of the model group (Fig. 5B-D).

The differences in the Glu/GABA ratios among groups in stages of NR1, R1 and R2 were analyzed by two-factor ANOVA (Fig. 6). In the NR1 stage, Glu/GABA ratios of the SYC and 
fluoxetine groups were significantly lower than those of the model group $(\mathrm{P}<0.05)$. In the $\mathrm{R} 1$ and $\mathrm{R} 2$ stage, there were no significant differences in Glu/GABA ratios among the five groups. The ratio of Glu to GABA in the model group was significantly lower than that in the control group during NR2 $(\mathrm{P}<0.01)$, and there was no significant difference between the two groups during NR1 (P>0.05). Glu/GABA ratios of the SYC and fluoxetine groups were significantly higher than those of the model group in NR2 $(\mathrm{P}<0.05)$; however, the Glu/GABA ratio of the SS group demonstrated no significant difference compared with the model group $(\mathrm{P}>0.05)$. These results suggested that the occurrence of PMS depression may be caused by an elevation in the Glu/GABA ratio and that SYC may effectively treat PMS depression by decreasing the Glu/GABA ratio.

\section{Discussion}

In the present study, a PMS depression model was established in female Wistar rats by chronic stress constraint, as previously described (31,32). Following this, the therapeutic effect of SYC on PMS was evaluated by body mass gain, OFT and SPT. OFT and SPT are classical behavioral evaluation methods for animal models of depression. In the OFT, the crossing score is mainly used for the evaluation of the animals' excitability, while the rearing score mainly assesses its interest and exploration of the surrounding environment. Therefore, the total scores of the OFT reflect comprehensive information, including tension, excitement, interest and adaptability (33). The core clinical symptom of depression is anorgasmia, which is characterized by a reduction in the sensitivity to reward and alterations to the reward level. A $1 \%$ sucrose solution is an effective reward to rats. $\mathrm{SC} \%$ is a common index for evaluating the degree of anhedonia in rats. For modeling, 30 female Wistar rats were treated by chronic stress constraint during two consecutive non-acceptance periods (four days) of the estrous cycle. Following four-day modeling, the total score of OFT and SC\% of SPT in the model group were all significantly lower than those of the control group. The total score of OFT and SC\% of SPT in the model group in the post-modeling period decreased significantly compared with those in the pre-modeling period. These results indicated that the PMS depression rat model was successfully established. Following four-day modeling, compared with the model group, the total score and SC\% of the SYC and fluoxetine groups increased significantly; however, there was no significant difference between the SYC and fluoxetine groups. These data illustrated that SYC was highly efficacious in improving the macroscopical behavior and relieving the symptoms of PMS depression in rats.

It has been widely acknowledged that PMS is triggered by hormonal events ensuing following ovulation. Compelling evidence has implicated a role for progesterone in the pathophysiology of PMS. However, rising concentrations of estradiol or a hormonal milieu consisting of estrogen alone, fails to trigger any of the symptoms that characterize PMS. It appears it is the neuroactive metabolites of progesterone that affect mood and behavior in PMS. In the brain, progesterone is metabolized to neuroactive steroids, which act as positive allosteric modulators of the GABA neurotransmitter system in the brain. GABA is a main inhibitory neurotransmitter that is widely distributed in the CNS and acts as an important regulator of negative emotion (34). It is derived from Glu by the decarboxylation of glutamic acid decarboxylase (GAD). Thus, it is possible that the Glu and GABA may have indispensable roles in the etiology of PMS.

Using a combination of microdialysis and HPLC analysis, the present study investigated the dynamic changes of Glu and GABA in the right hippocampus of rats with PMS depression. The most robust result in the present study was that the ratio of Glu to GABA in the right hippocampus of rats with PMS depression decreased distinctly in the non-acceptance phase of the estrous cycle and that this decrease was reversed by SYC and fluoxetine treatment. These data further suggest edthat SYC and fluoxetine were not only effective in improving macro-behavioral indicators (OFT and SPT), but also in regulating pathological changes of micro-central indicators (Glu and GABA).

PMS is characterized by symptoms involving body, mind and behavior that emerge cyclically one to two weeks prior to menstruation (35). These symptoms are relieved soon following the onset of menses. Therefore, it is crucial and necessary to study the changes of Glu and GABA levels in different estrous cycles. Our research team has been engaging in clinical and experimental studies on PMS for decades, and previous studies have demonstrated that neurotransmitters, including 5-HT, NE and GABA, were one of the etiological factors of PMS (36-38). However, these studies were conducted in vitro with brain tissues or blood, and were unable to examine the dynamic changes in neurotransmitter levels that were observed in vivo. In the present study, in vivo levels of Glu and GABA in different phases of the estrous cycle in rats with PMS depression were detected. The NR1 and NR2 phases of rats in the present study were equivalent to the premenstrual phase of normal females and those with PMS, respectively. Data analysis demonstrated that the Glu levels were inconsistent with the macroscopic behavior. By contrast, the recorded expression levels of GABA were consistent with the macroscopic behavior. Therefore, it was presumed that the conclusion would be biased if the evaluation was based on the changes of Glu or GABA alone.

There is a delicate excitation/inhibition balance between the two neurotransmitters, Glu and GABA, in the CNS (22). In the present study the ratio of Glu to GABA in the hippocampus of rats with PMS depression was investigated. Two-factor ANOVA demonstrated that the Glu/GABA ratio of the model group in NR2 was significantly lower than that of the NR1 stage and that of the control group in NR2 stage. These results suggested that one of the mechanisms of PMS depression may be the depletion of the Glu/GABA ratio in the hippocampus and that the delicate excitation/inhibition balance may be disrupted in PMS depression. In addition, the Glu/GABA ratio of the SYC and fluoxetine groups were significantly higher than that of the model group in the NR2 phase, suggesting that SYC and fluoxetine were effective in restoring the excitation/inhibition balance in PMS depressive rats. Therefore, this may be one of the critical mechanisms underlying the therapeutic effect of these two drugs in PMS depression. By contrast, saikosaponin extracted from bupleurum chinense demonstrated a weak effect on PMS, suggesting that the prescription of Traditional Chinese Medicine was scientific and the compatibility was necessary.

In conclusion, the results of the present study demonstrated that chronic stress constraint during two successive non-acceptance phases of the estrous cycle successfully induced a PMS 
depression model in rats. The ratio of Glu to GABA in the right hippocampus of the model rats was significantly lower than that of the normal rats. Furthermore, the novel Chinese Medicine preparation SYC significantly improved the microscopic behavioral indicators (OFT and SPT) and inhibited the decrease of the Glu to GABA ratio. Therefore, it is concluded that the underlying therapeutic effect of SYC in PMS depression may involve the maintenance of the excitation/inhibition balance between Glu and GABA in the CNS.

\section{Acknowledgements}

This study was supported by the National Natural Science Foundation of China (Key Program; nos. 30930110, 30973688, 81173151, 81102537 and 81173163).

\section{References}

1. Yonkers KA, O'Brien PM and Eriksson E: Premenstrual syndrome. Lancet 371: 1200-1210, 2008.

2. Shah NR, Jones JB, Aperi J, Shemtov R, Karne A and Borenstein J: Selective serotonin reuptake inhibitors for premenstrual syndrome and premenstrual dysphoric disorder: a meta-analysis. Obstet Gynecol 111: 1175-1182, 2008.

3. Brown J, O'Brien PM, Marjoribanks J and Wyatt K: Selective serotonin reuptake inhibitors for premenstrual syndrome. Cochrane Database Syst Rev 15: CD001396, 2009.

4. Biggs WS and Demuth RH: Premenstrual syndrome and premenstrual dysphoric disorder. Am Fam Physician 84: 918-924, 2011.

5. Nijenhuis CM, Horst PG, Berg LT and Wilffert B: Disturbed development of the enteric nervous system after in utero exposure of selective serotonin re-uptake inhibitors and tricyclic antidepressants. Part 1: Literature review. Br J Clin Pharmacol 73: 16-26, 2012.

6. Sansone RA and Sansone LA: SSRIs: bad to the bone? Innov Clin Neurosci 9: 42-47, 2012.

7. Tatara A, Shimizu S, Shin N, Sato M, Sugiuchi T, Imaki J and Ohno Y: Modulation of antipsychotic-induced extra pyramidal side effects by medications for mood disorders. Prog Neuropsychopharmacol Biol Psychiatry 38: 252-259, 2012.

8. Tan Q and Zhang HY: Effect of shuyu capsule on location and expression of 5-hydroxy tryptamine3B receptor in hippocampus and hypothalamus regions in rats with depression emotion. Chinese Journal of Experimental Traditional Medical Formulae 17: 137-139, 2011.

9. Jiang YF, Gao J, Wei S, Xue L and Ge QF: Effect of shuyu capsule contained serum on the expression of $\gamma$-aminobutyric acid B2 receptor in primary cultured rat hippocampal neurons. Chinese Journal of Experimental Traditional Medical Fomulae 18: 165-169, 2012.

10. Allen LM and Lam AC: Premenstrual syndrome and dysmenorrhea in adolescents. Adolesc Med State Art Rev 23: 139-163, 2012.

11. Pereira-Vega A, Sánchez Ramos JL, Vázquez Oliva R, et al: Premenstrual asthma and female sex hormones. J Investig Allergol Clin Immunol 22: 437-439, 2012.

12. Rapkin AJ and Akopians AL: Pathophysiology of premenstrual syndrome and premenstrual dysphoric disorder. Menopause Int 18: 52-59, 2012.

13. Wilson CL, Maidment NT, Shomer MH, Behnke EJ, Ackerson L, Fried I and Engel J Jr: Comparison of seizure related amino acid release in human epileptic hippocampus versus a chronic, kainate rat model of hippocampal epilepsy. Epilepsy Res 26 : 245-254, 1996

14. Aguilera A, Selgas R, Codoceo R and Bajo A: Uremic anorexia: a consequence of persistently high brain serotonin levels? The tryptophan/serotonin disorder hypothesis. Perit Dial Int 20: $810-816,2000$

15. Mitchell ND and Baker GB: An update on the role of glutamate in the pathophysiology of depression. Acta Psychiatr Scand 122: 192-210, 2010

16. Mueller PJ and Mischel NA: Selective enhancement of glutamate-mediated pressor responses after GABA(A) receptor blockade in the RVLM of sedentary versus spontaneous wheel running rats. Front Physiol 3: 447, 2012.
17. Crabtree JW, Lodge D, Bashir ZI and Isaac JT: GABAA, NMDA and mGlu2 receptors tonically regulate inhibition and excitation in the thalamic reticular nucleus. Eur J Neurosci 37: 850-859, 2013.

18. Batra NA, Seres-Mailo J, Hanstock C, et al: Proton magnetic resonance spectroscopy measurement of brain glutamate levels in premenstrual dysphoric disorder. Biol Psychiatry 63: 1178-1184, 2008.

19. Krystal JH, Sanacora G, Blumberg H, et al: Glutamate and GABA systems as targets for novel antidepressant and mood-stabilizing treatments. Mol Psychiatry 7 (Suppl 1): S71-80, 2002.

20. Kendell SF, Krystal JH and Sanacora G: GABA and glutamate systems as therapeutic targets in depression and mood disorders. Expert Opin Ther Targets 9: 153-168, 2005.

21. Zorumski CF, Paul SM, Izumi Y, Covey DF and Mennerick S: Neurosteroids, stress and depression: potential therapeutic opportunities. Neurosci Biobehav Rev 37: 109-122, 2013.

22. Konradi C and Heckers S: Molecular aspects of glutamate dysregulation: implications for schizophrenia and its treatment. Pharmacol Ther 97: 153-179, 2003.

23. Epperson CN, Haga K, Mason GF, et al: Cortical gamma-aminobutyric acid levels across the menstrual cycle in healthy women and those with premenstrual dysphoric disorder: a proton magnetic resonance spectroscopy study. Arch Gen Psychiatry 59: 851-858, 2002.

24. Duman RS, Malberg J, Nakagawa S and D'Sa C: Neuronal plasticity and survival in mood disorders. Biol Psychiatry 48: 732-739. 2000.

25. Bäckström T, Haage D, Löfgren M, et al: Paradoxical effects of GABA-A modulators may explain sex steroid induced negative mood symptoms in some persons. Neuroscience 191: 46-54, 2011.

26. Zhang HY, Wei S, Sun P, Xue L and Qiao MQ: Empirical study of changes of pre-menstrual syndrome model rats with liver-qi invasion and liver-qi depression in peripheral blood, sexual hormones, different encephalic regions and accommodate hormones. World Science and Technology 12: 51-56, 2010.

27. Shigemi K, Tsuneyoshi Y, Yamada S, Kabuki Y, Hayamizu K, Denbow DM and Furuse M: Oral administration of L-serine reduces the locomotor activity of socially isolated rats. Neurosci Lett 468: 75-79, 2010

28. Rygula R, Abumaria N, Havemann-Reinecke U, et al: Pharmacological validation of a chronic social stress model of depression in rats: effects of reboxetine, haloperidol and diazepam. Behav Pharmacol 19: 183-196. 2008.

29. Pandey DK, Mahesh R, Kumar AA, Rao VS, Arjun M and Rajkumar R: A novel 5-HT(2A) receptor antagonist exhibits antidepressant-like effects in a battery of rodent behavioural assays: approaching early-onset antidepressants. Pharmacol Biochem Behav 94: 363-373, 2010.

30. Paxinos G and Watson C (eds): The Rat Brain in Stereotaxic Coordinates-The New Coronal Set. 5th edition. Elsevier Academic Press, San Diego, CA, pp27, 2005.

31. Ho HP, Olsson M, Westberg L, Melke J and Eriksson E: The serotonin reuptake inhibitor fluoxetine reduces sex steroid-related aggression in female rats: an animal model of premenstrual irritability? Neuropsychopharmacology 24: 502-510, 2001.

32. Smith SS, Ruderman Y, Hua Gong Q and Gulinello M: Effects of a low dose of ethanol in an animal model of premenstrual anxiety. Alcohol 33: 41-49, 2004

33. Võikar V, Rauvala H and Ikonen E: Cognitive deficit and development of motor impairment in a mouse model of Niemann-Pick type C disease. Behav Brain Res 132: 1-10, 2002.

34. Rupprecht R: Neuroactive steroids: mechanisms of action and neuropsychopharmacological properties. Psychoneuroendocrinology 28 139-168, 2003.

35. Cunningham J, Yonkers KA, O'Brien S and Eriksson E: Update on research and treatment of premenstrual dysphoric disorder. Harv Rev Psychiatry 17: 120-137, 2009.

36. Qiao M,Zhao Q, Zhang H, Wang H, Xue L and Wei S: Isolating with physical restraint low status female monkeys during luteal phase might make an appropriate premenstrual depression syndrome model. J Affect Disord 102: 81-91, 2007.

37. Qiao M, Zhang H, Yu Y, Ci Y, Xu X, Ye Q and Chen Y: Dynamic changes in serum estradiol and progesterone levels in patients of premenstrual syndrome with adverse flow of liver-qi. J Tradit Chin Med 28: 106-109, 2008.

38. Qiao M,Zhang H,Liu H, et al: Prevalence of premenstrual syndrome and premenstrual dysphoric disorder in a population-based sample in China. Eur J Obstet Gynecol Reprod Biol 162: 83-86, 2012. 